\title{
2008 International Materials Research Conference, Beijing AAA Satellite Meeting Held in June
}

\author{
http://ccmst.hit.edu.cn/2008Meeting
}

The 2008 International Materials Conference, Beijing AAA Satellite Meeting on "Advanced Technologies for Advanced Characterizations of Advanced Materials," endorsed by the Materials Research Society (MRS) and sponsored by the Chinese Materials Research Society (CMRS), was held at the Institute of Physics (IOP) of the Chinese Academy of Sciences (CAS), in Beijing, China, on June 15-18, 2008. The meeting was organized by Yusheng Zhao (Los Alamos National Laboratory, USA), Quanxi Jia (Los Alamos National Laboratory, USA), Changqing Jin (Institute of Physics, CAS, China), and Haozhe Liu (Harbin Institute of Technology, China).

On behalf of the organization committee, Zhao opened the meeting with a brief introduction, welcoming the audience of about 70 scientists from 11 countries. About half of the attendees were young students and post-doctorates. Over 30 oral presentations and about 20 posters were presented at the meeting. The Satellite Meeting was fittingly held at IOP/CAS which is celebrating its 80th anniversary. The IOP deputy director Hongjun Gao spoke briefly to welcome all the meeting attendees and introduced the pathway and great achievements of IOP over the past 80 years.

The goal of the meeting was to provide a forum for researchers and scientists in the fields of advanced materials and advanced technologies to discuss their latest research results, exchange ideas, and promote interdisciplinary research. The Meeting encouraged presentations in all research aspects of advanced materials, such as processing, modeling, and characterization. Furthermore, the Meeting encourages international

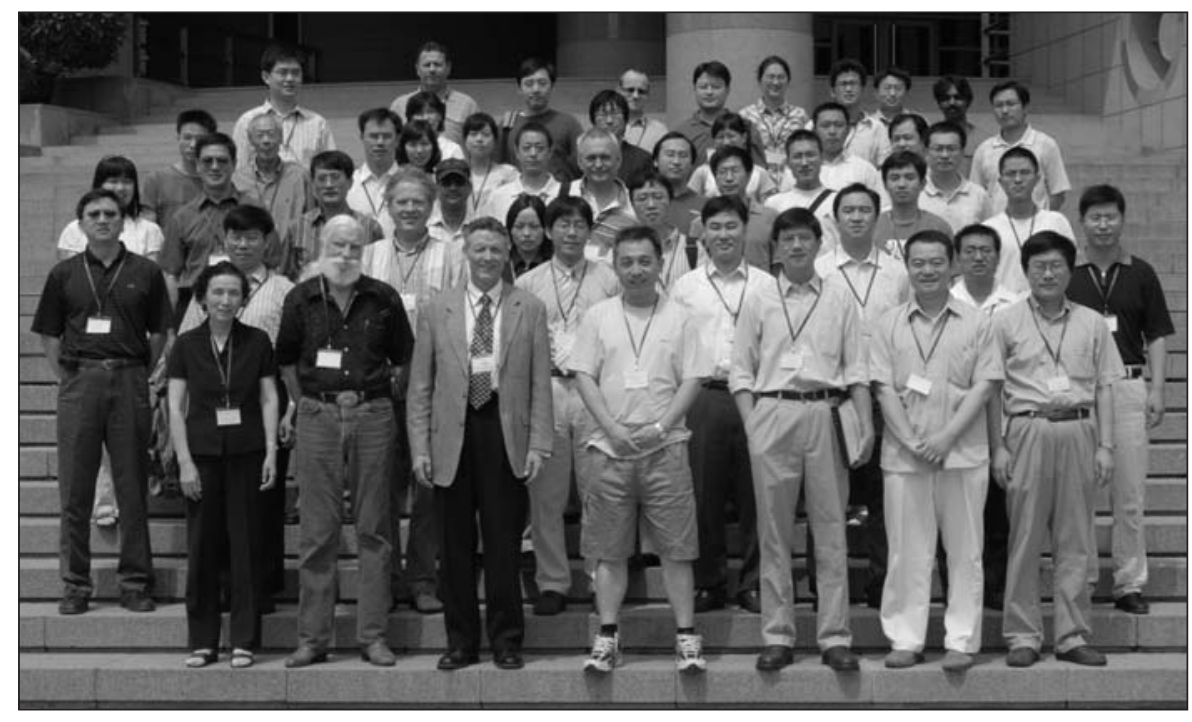

Participants of the 2008 International Materials Research Conference, Beijing AAA

Satellite Meeting met on June 15-18, 2008, at the Chinese Academy of Sciences' Institute of Physics.

collaboration with researchers in China. Invited talks covered a broad range of state-of-the-art materials science research, including nanomaterials science and technology (Z. Wang, Georgia Inst. of Technology, USA; H. Gao, IOP/CAS, China; Z. Liu, Peking University, China; and Y. Zhang, Intel Corp., USA) and superconductivity and magnetoelectricity (P. Dai, Oak Ridge National Laboratory, USA; Y. Shimakawa, Kyoto University, Japan; K.-H. Kim, Seoul National University, South Korea; and P.M. Grant, Stanford University, USA). Further presentations were given on in situ electron microscopy and neutron/ synchrotron dif- fraction, the growth of thin-film and interface characterization, and ultrasonic and high-pressure techniques.

Alan J. Hurd of Los Alamos National Laboratory and the immediate past president of MRS gave the plenary presentation on the global direction of materials research. He particularly encouraged new studies on energy and environmental materials, topics currently receiving urgent attention from MRS.

The Institute of Physics, CAS, provided the meeting hall and accommodation and the Harbin Institute of Technology provided partial financial support.

\section{Advertisers in This Issue}

\begin{tabular}{|c|c|c|c|}
\hline & Page No. & & Page No. \\
\hline Agilent Technologies & 813 & Huntington Mechanical Laboratories, Inc. & Outside back cover \\
\hline AJA International, Inc. & 846 & Janis Research Company, Inc. & 845 \\
\hline Arizona State University 2009 Winter School & 845 & JEOL USA Inc. & 810 \\
\hline Bruker AXS Inc. & Inside back cover & Nature Publishing Group & 817 \\
\hline High Voltage Engineering Europa B.V. & Inside front cover & Veeco Instruments Inc. & 809 \\
\hline Hindawi Publishing Corp. & 814 & J.A. Woollam Co, Inc. & 871 \\
\hline
\end{tabular}

For free information about the products and services offered in this issue, check http://www.mrs.org/bulletin_ads. 